\title{
ICMJE statement on compulsory clinical trial registration: should Indian journals follow suit?
}

\author{
GITANSALI BATMANABANE
}

Professor of Pharmacology, Jawaharlal Institute of Postgraduate Medical Education and Research (JIPMER), Pondicherry, Associate Editor, Indion Joumal of

Pharmacology. Address for correspondence: Department of Pharmacology, JIPMER, Pondicherry 605006 INDIA. e-mail: gitanjali@jipmer.edu

In May 2005, the International Council for Medical Journal Editors (ICMJE), a group of general medical journal editors who have been responsible for introducing the "uniform requirements for manuscripts submitted to biomedical journals", released a joint editorial statement on registration of clinical trials (1). ICMJE journals will consider manuscripts reporting clinical trials only if the trial was registered in a public registry before patient recruitment started. Trials started before the ICMJE statement have been given a grace period within which they must be registered. This is an update of the ICMJE statement published a few months earlier (2). The statements are a first step to regulate and make transparent the messy and murky world of clinical trials.

The ICMJE seeks to create an accessible and searchable public record of all clinical trials, containing all the relevant information clinicians need to make informed decisions regarding a particular treatment option, not just what "the researchers wanted to report and the editors chose to publish"(1)

The ICMJE has clarified that registration is required of alf "clinically directive trials" - phase 3 trials that test a health outcome, whether drug, surgical procedure, behavioural therapy or screening procedure. In short, any study where an intervention is compared with a concurrent control group. Among those exempted trials are the purely pharmacokinetic studies or phase 1 clinical trials. However, the ICMJE urges researchers, when in doubt, to go ahead and register.

The ICMJE statement was signed by editors representing major international medical journals with the exception of the BMJ which supported the idea but differed on the type of trial registry $(3,4)$.

The idea of registering clinical trials, mooted nearly three decades ago, has long been resisted. The example of paroxetine illustrated the importance of early registration and transparency in clinical trials. Certain safety and efficacy data of paroxetine in major depressive disorders in children and young adults (5) had been withheld from publication. As a result, thousands of patients were prescribed the drug unnecessarily. This is just one example of a widespread problem.

\section{Clinical trials in India}

India is currently touted as a haven for clinical trials; it is projected that by 2010 clinical trials to the tune of $\$ 250-300$ million annually will be conducted in India (6). In the absence of a national registry of clinical trials Indian researchers and the public may not know what studies are being conducted on Indian soil, by whom and where. This might result in duplication of work and wastage of resources. It could expose research participants to unnecessary risks. National funding agencies may sanction money for similar studies, unaware that such work is underway or has already been done, since adverse or negative results may not get published at all. Finally, it permits human research to be done without public scrutiny.

\section{Clinical trial reports published in Indian journals}

Trials supported by the pharmaceutical industry and conducted in India get published in international journals - or in house journals for distribution to doctors. It is my impression that Indian journals publish a dismally small percentage of those clinically directed (phase 3 ) studies conducted in this country. The few studies that do get published here are usually academic dissertations of postgraduate medical students. These are generally called 'pilot' studies though in reality there is no full study in progress. Studies undertaken with inadequate sample sizes are reported as 'preliminary' when there are no further results to publish. Poorly planned and conducted studies eventually get published in non-peer-reviewed journals.

Authors working in India prefer to submit their betterwork to an international journal when their work reveals important conclusions. Reports published in Indian medical journals are usually of lesser therapeutic impact.

The situation is unlikely to change with the ICMJE statement. One might argue that if Indian medical journals do not insist on prior registration, our journals will attract trial reports which would have otherwise gone to North American and British journals. However, it is unlikely that clinicians in the West will start submitting reports to Indian journals. Pharmaceutical companies will still want their trials to be published in western journals for the impact it will have on the sale of their products. 


\section{Mandatory registration in India}

However, if clinical trial registration becomes mandatory in India, it could have an impact on the quality of research done here. It might initially dampen the enthusiasm of medical students but it would eventually elevate the standard of research as researchers would be forced to plan better studies since their protocols would be open to criticism. The number of poorly designed studies will certainly come down if ICMJE criteria are followed; these criteria include publication of the entire protocol, including sample size, before recruitment of the first subject begins (1). This will force the academic and moral introspection necessary to improve standards of research in medical colleges.

The ICMJE statement also insists on ethics committee clearance for these trials (1). Perhaps Indian medical journal editors could do what the Indian Council of Medical Research (ICMR) has been unable to do; the ICMR's ethical guidelines on biomedical research (7) have not prevented the conduct of clinical trials without ethics committee clearance. The mistaken notion that only clinical trials with a drug require ethical clearance has permitted thousands of studies testing new kits, psychotherapy, interventions using implants, surgery etc., being done in medical colleges and other institutions all over India without any research ethics review (8).

In fact the ICMJE statement could be accepted not just by editors but also as a prerequisite for funding by national agencies, award of degrees (if the trial formed part of a thesis) and even presentation at conferences.

A public repository could be maintained of the kind being promoted in the US (2) supported by all major funding bodies. All trials funded by national agencies should be registered here. Public funds are used for clinical trials on Indian systems of medicine and the public have a right to know about them.

What if trials are conducted in India and not published? Trials banned in other countries for safety and ethical reasons may be done here, to generate data for the data banks of pharmaceutical companies. The ICMJE statement does not address this problem.

Still, all editors of medical journals published from India should come forward and endorse this statement which intends to promote transparency in the conduct of clinical trials. This can only become a reality when all journals insist on it. At stake is the altruism of thousands of Indian men and women volunteering for these trials.

\section{References}

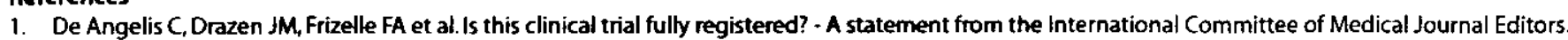
(editorial) NEngl J Med 2005;352:2436-7.

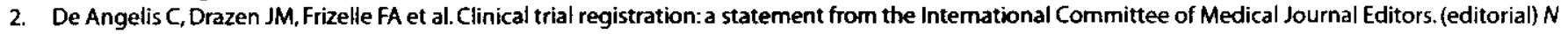
EnglJ Med 2004;351:1250-1.

3. Abbasi K Compulsory registration of clinical trials. BMJ 2004;239:637-8.

4. Abbasi K, Godlee F. Next steps in trial registration Minimum criteria have been agreed, and intentions restated. BM $2005 ; 330: 1222-1223$.

5. Rennie D. Trial registration. A great idea switches from ignored to irresistible. JAMA 2004; 292:1359-62.

6. Bhatt A. Clinical trials in India: Pangs of globalization. Indian J Pharmacol 2004;36:207-8.

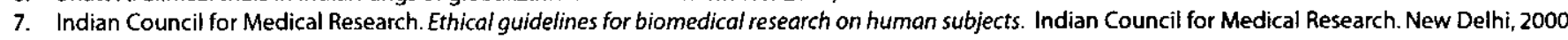
http://icmr.nic.in/ethical.pdf (accessed June 9, 2005).

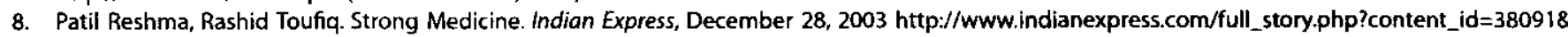
spf=true (accessed on June 15, 2005). 